\title{
Gustav Ritter von Wex, sein Leben und Werk
}

Zusammenfassung: Die Donau-Regulierung entlang der Stadt Wien um 1870 wird als eines der großen europäischen Flussbauprojekte des 19. Jahrhunderts beschrieben. Dabei werden insbesondere Publikationen des Projektleiters Gustav von Wex (1811-1892) verwendet, da dieser die geschichtlichen Grundlagen, den kaiserlichen Entscheid, das Bauprojekt und die Baufortschritte ausführlich beschrieben hat. Weiter werden auch zusätzliche wissenschaftliche Arbeiten von Wex' diskutiert und in einen historischen Kontext gestellt. Schließlich wird die Biographie von Wex' umrissen und die Probleme bei der Aufsuchung seines Portraits dargestellt. Mit dieser historischen Arbeit soll ein Beitrag zu den frühen Entwicklungen des europäischen Flussbaus geleistet werden.

Gustav Ritter von Wex, his life and work

Summary: The regulation works of Danube river along Vienna city around 1870 are described as a monumental European project of the 19th century. The publications of the project head Gustav von Wex (1811-1892) are thereby reviewed, given that he therein considered the historical bases, the imperial decision, the project outline and the advances of the works. In addition, the scientific works of von Wex are also described and put into a historical context. Finally, the biography of von Wex is presented along with the problems in searching successfully his portrait. This historical work is intended to add to the early developments in river engineering of Europe.

\section{Einleitung}

Die Donau fließt beim Leopoldsberg aus einem engen Bett um dann in das Wiener Becken zu strömen. Dadurch haben sich im Verlauf der Zeit in diesem Becken Sedimente abgelagert. Infolge der reduzierten Fließgeschwindigkeit kam es zudem bei Hochwasser zu Überflutungen und Eisgängen, von denen die Hauptstadt oft

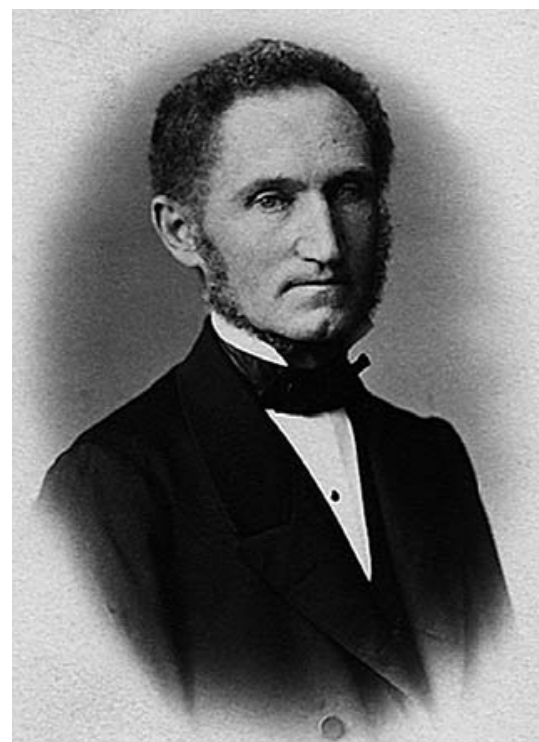

Abb. 1: : Gustav Ritter von Wex um 1880 (ÖIAV 2003)

bedroht wurde. So forderte etwa 1830 ein verheerender Eisstau viele Opfer in Wien, sodass die Forderung der Bevölkerung nach einem entsprechenden Schutz vor der Donau immer lauter wurde. Erst nach der Tauflut von 1862 wurde ein Entschluss gefasst, der dann die große Regulierung der Donau um die Stadt Wien nach sich zog (Abb. 2). Mit ,kaiserlicher Entschließung' wurde 1864 eine Studienkommission beauftragt, diese Regulierungsarbeiten zu untersuchen. Nach vier Jahren kam das international zusammengesetzte Gremium zu folgenden Resultaten: (1) Zwischen Nussdorf und Albern sollte ein neues Flussbett geschaffen werden mit einem $285 \mathrm{~m}$ breiten und $3.15 \mathrm{~m}$ tiefen Mittelwasserbett und einem $440 \mathrm{~m}$ breiten Hochwasserbett, (2) Am linken Flussufer soll ein Damm errichtet werden, während am rechten Ufer die Anlagen der Schifffahrt erstellt werden sollten, und (3) Alle Donauarme mit Ausnahme der Donaukanals sind abzubauen. Am Eintritt des Donaukanals ist ein Hochwasserabschluss zu erstellen. Die Kosten des Projekts wurden 1868 auf 24.6 Mio. damalige Gulden veranschlagt.

Die Arbeiten begannen um 1870 , am 14. April 1875 wurde das Wasser in den neuen Durchstich eingeleitet und am 30. Mai 1875 erfolgte die feierliche Eröffnung des Werks durch den Kaiser. Die Vollendungsarbeiten währten bis 1884 und erforderten weitere 6 Mio. Gulden. Die Vorteile, welche die Donauregulierung insbesondere hinsichtlich Hochwasserschutz und Binnenschifffahrt bot, wurden erst dann vol wirksam. Etwa zur selben Zeit wurde auch die Regulierung der gesamten Donaustrecke von der Isper-Mündung bis zur ungarischen Grenze genehmigt, welche dann kurz vor Beginn des 1. Weltkriegs fertig gestellt wurde. Es soll hier vielleicht noch festgehalten werden, dass die Donau bei Wien einen historischen Maximaldurchfluss von rund $14000 \mathrm{~m}^{3} / \mathrm{s}$ im Jahre 1501 gehabt haben soll.

Die Donau-Regulierungs-Kommission wurde 1868 ins Leben gerufen aber 1927 wieder aufgelöst, da dann deren Arbeiten vom Bundesstrombauamt in Wien übernommen wurden, dessen Wirkungskreis sich auf die ganze österreichische Donau und das internationale Donaunetz erstreckte. Von bleibendem Wert dieser Arbeiten sind die gleichmäßige Vertiefung und Verbreiterung der Flussstrecke zu erwähnen, jedoch führten die Vorkehrungen am oberen Ende des Donaukanals mit den damaligen Mitteln zu keinem befriedigenden Ergebnis. Erst im 20. Jahrhundert wurde dort mittels eines Schwimmtors die Hochwassergefahr von den Ufergebieten des Donaukanals einigermaßen abgehalten. Weitere Arbeiten erfolgten im Zuge der Ausdehnung der Stadt Wien, die hier aber nicht weiter besprochen werden sollen.

Im folgenden soll die historische Entwicklung der Donau-Regulierung hauptsächlich anhand der Publikationen von Wex' erläutert werden und das gesamte Projekt in einen internationalen Vergleich gestellt werden. Es handelte sich in der Tat um eines der grössten Wasserbauprojekte während der 2. Hälfte des 19. Jahrhunderts. Parallel dazu soll auch die Person von Wex vorgestellt werden, da er einen massgebenden Anteil am Projekt und an dessen Gelingen hatte. 


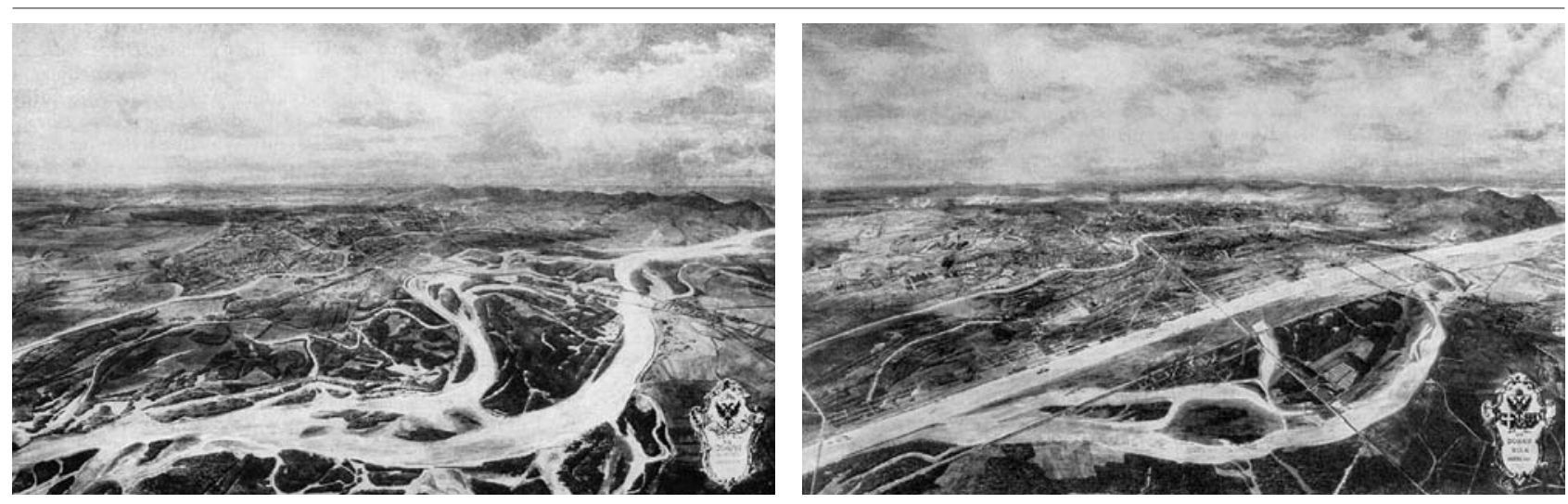

Abb. 2: : Die Donau bei Wien (a) vor und (b) nach der Regulierung (Künster und Jarosch 1935)

\section{Biografie}

Gustav Wex wurde in Neumarkt (heute Nowy Targ bei Krakau, Polen), damals im österreichischen Galizien, am 13.10.1811 geboren. Nachdem er seine dreijährigen Ingenieurstudien an der TU Wien 1831 beendet hatte, trat er in das k.k. Öffentliche Amt für Arbeit ein, wo er sein weiteres Berufsleben verblieb. Er fungierte als Chef des Technischen Departements der Niederösterreichischen Statthalterei, als das Projekt der Donau-Regulierung am 12.09.1868 genehmigt und er mit der Durchführung der Vorarbeiten sowie mit der Oberleitung des Baus betraut wurde. Seine hervorragenden und verdienstvollen Leistungen in diesem Projekt wurden mit Auszeichnungen reichlich belohnt (Abb.1). 1880 trat von Wex in den Ruhestand, ohne jedoch das Interesse an seinem begonnenen grossen Projekt und am Wasserbau generell zu verlieren. Neben seinen Projekten hat von Wex auch reichlich in den Fachjournalen sowohl über die Donau-Regulierung als auch über andere Aspekte des Fluss- und Wasserbaus berichtet. Dabei hat er dem Österreichischen Ingenieur- und Architekten-Verein während Jahren als treues und engagiertes Mitglied gedient. Dadurch war er auch als Experte, beispielsweise an der Wiener Hochquellenleitung in den 1860er Jahren oder in der Umgestaltung des Wiener Donaukanals um 1890 tätig. Er war weit über sein Land hinaus als Wasserbautechniker bekannt und geschätzt. von Wex schied nach längerem Leiden am 26.09.1892 in Bad Ischl dahin und wurde zu Weidling bei Klosterneuburg bestattet (Anonym 1892, Roeder 1892, Kleinschroth 1992).

1876 wurde dem k.k. Hofrath und Oberbauleiter der Donau-Regulierung bei Wien, Herrn Gustav Ritter von Wex, das Offizierskreuz des St. Mauritius- und Lazarus-Ordens verliehen (Anonym 1876).
Kurz vorher wurde er vom Kaiser in den Ritterstand erhoben. 1881 wurde Ritter von Wex in Würdigung seiner Leistungen auf dem Gebiete der Hydrotechnik vom König Italiens das Gross-Offizierskreuz des Ordens der Italienischen Krone allergnädigst verliehen (Anonym 1881).

Die Suche des Portraits von Wex' gestaltete sich mühsam. Ein erster Kontakt mit der TU Wien im Jahr 2000 erwies sich als negativ, obwohl ich von dort bereits einige wertvolle Unterlagen erhalten habe. Bei einer Nachfrage am Österreichischen Staatsarchiv wurde mir empfohlen, das Bildarchiv der Österreichischen Nationalbibliothek am Heldenplatz, Wien, zu kontaktieren, leider wiederum erfolglos. Weiter ging es an das Magistrat der Stadt Wien, wo man mir wiederum nicht behilflich sein konnte, mich jedoch an das Archiv der TU Wien am Karlsplatz verwies, wo das Portrait ebenfalls nicht zu finden war. Nebenbei wurde mir eine Todesanzeige vom Hydrauliker Romuald Iszkowski (1848-1904) beigelegt, des Schwiegersohns von Wex'. Meine Portraitsuche ging weiter zur Wiener Staats- und Landesbibliothek, Wiener Rathaus: kein Resultat, zur Wiener Zeitung und zur Kronen-Zeitung: kein Resultat, nach Weidling zum Kath. Pfarramt: kein Resultat, zum Wiener Landesarchiv, zum Illustrierten Wiener Extrablatt und schliesslich zur Heraldisch-Genealogischen Gesellschaft „Adler": Wiederum kein Resultat! Auch bei weiteren historischen Zeitschriften wurde ich leider nicht fündig. Auf Internet-Seiten betreffend der Donau-Regulierung war auch kein Portrait von Wex' zu finden.

Von der Wiener-Zeitung erhielt ich Briefpost. Der Verfasser des Briefs war im Wiener Staatsarchiv, hat dort aber ebenfalls nichts gesichtet. Er lud mich sogar ein, ihm einen Artikel über von Wex zu senden. Normalerweise hätte jeder die Suche schon lange abgebrochen, ich machte weiter, wie schon viele Male, meistens mit Erfolg. Beim Österreichischen Staatsarchiv, Abteilung Kriegsarchiv, erhielt ich ebenfalls eine Absage; bei der Abteilung Allgemeines Verwaltungsarchiv teilte man mir mit, dass ich zur besseren Einsicht in die Aktenlage die entsprechenden Seiten kopieren lassen sollte, inklusive einer Farbkopie des Familienwappens, mit Kosten inkl. der Auslandsportospesen von 230,- öSchilling. Da ich nur das Portrait suchte, sagte ich ab. Weitere Antworten trafen 20 Monate nach Suchbeginn wieder von der TU Wien ein, wo man mir einen (bereits bekannten) Nachruf vorlegte, kurz später sagte man mir auch, dass in der Österreichischen Wochenschrift für den öffentlichen Baudienst des Jahres 1892 (der an der ETH-Bibliothek Zürich fehlt) leider ebenfalls kein Nekrolog wäre. Das Magistrat Wien teilte mir dann mit, dass eine einzige Person noch Zugriff zu den alten Unterlagen hätte, dieser wäre trotz Pensionierung bereit, die Suche weiter voranzutreiben. Sein Bericht war schließlich aber ebenfalls negativ.

Dann ging ich nochmals zurück zum Anfang und nahm mit dem Österreichischen Ingenieur- und Architekten-Verein Kontakt auf. Vorerst teilte man mir mit, dass es in Wien eine Wexstraße am Leipziger Platz gäbe. Schliesslich nahm ich man glaube es oder nicht - mit einem Martin Wex Kontakt auf, der mir aber mitteilte, nachdem er als Tiroler alle seine Ahnenpapiere durchsucht hatte, keinen Gustav gesichtet hätte. Eine Telefonsuche aller Wex Österreichs - insgesamt 43 Personen, war ebenfalls nicht erfolgreich. Zu dieser Zeit hatte ich als Schweizer offensichtlich noch nicht realisiert, dass von Wex' Geburtsort Neumarkt nicht am Wallersee in Österreich, sondern im heutigen Polen war, denn eine Nachforschung des Pfarrers vom heute österreichschen Neumarkt war ebenfalls von keinem Erfolg gekrönt. 
Weiter ging's nach Bad Ischl, wo man mir den Nekrolog von Wex' aus der Ischler Wochenschau zusandte, ohne Portrait wohlverstanden. Weitere negative Nachrichten trafen von der Österreichischen Nationalbibliothek, Bildarchiv und Portraitsammlung anfangs 2003 ein. Ich erhielt dann auch eine E-Mail aus Nowy Targ, dem Geburtsort von Wex im heutigen Polen. Man teilte mir mit, dass Gustaw Wex, Sohn von Wilhelm Wex und Anna Kurner am 13.10.1811 dort geboren worden wäre, ein Datum, das ich bis anhin noch nicht gekannt hatte. Das Landesmuseum Joanneum, das ich auf Anraten ebenfalls kontaktiert hatte, konnte mir hingegen nicht helfen. Und dann, im Oktober 2003, erhielt ich eine Mail vom ÖIAV, Wien, welcher ein Portrait von Wex' beilag (Abb. 1). Ich hatte die aktive Suche schon länger aufgegeben, mein Buch „Hydraulicians in Europe" war kurz vorher publiziert worden, ohne das Portrait von Wex zu enthalten. Immerhin, die lange Suche hat sich schliesslich gelohnt, und das Portrait liegt dieser Publikation bei. Man teilte mir auch mit, dass von Wex bis 1890 an der Johannesgasse 14 in Wien I gewohnt hatte.

\section{Publikationen}

\subsection{Donau}

In seiner ersten Publikation bespricht der damalige Ministerial-Oberingenieur von Wex (1857) die Hindernisse auf der Donau in den beiden Donau-Fürstentümern Moldau und Walachei. Im Nachgang zu den Kriegen des Jahres 1853 wurde die Donau dort blockiert, womit eine geregelte Schifffahrt verunmöglicht wurde. Diesem misslichen Zustand machte die österreichische Regierung dadurch ein Ende, dass sie die territoriale Autorität am Fluss Sulina übernahm um so die Schifffahrt auf der unteren Donau gemäß dem Pariser Frieden zu ermöglichen. von Wex beschreibt die Hindernisse durch die am Eisernen Thore gelegenen Stromschwellen, welche insbesondere bei Niedrigwasser die Schifffahrt stark behinderten. An der unteren Donau sollen in diesen Jahren rund 2500 Seeschiffe beobachtet worden sein, welche einen bedeutenden Handeln zwischen den wichtigsten Donau-Städten sicherstellten. von Wex plädiert schliesslich für die Erbauung des Sulina-Kanals, eines Wasserweges also, der im heutigen Tulcea (Rumänien) die Donau Richtung Osten verlässt, um bei Sulina direkt in das Schwarze Meer zu münden. Damit ließe sich die Untere Donau besser für

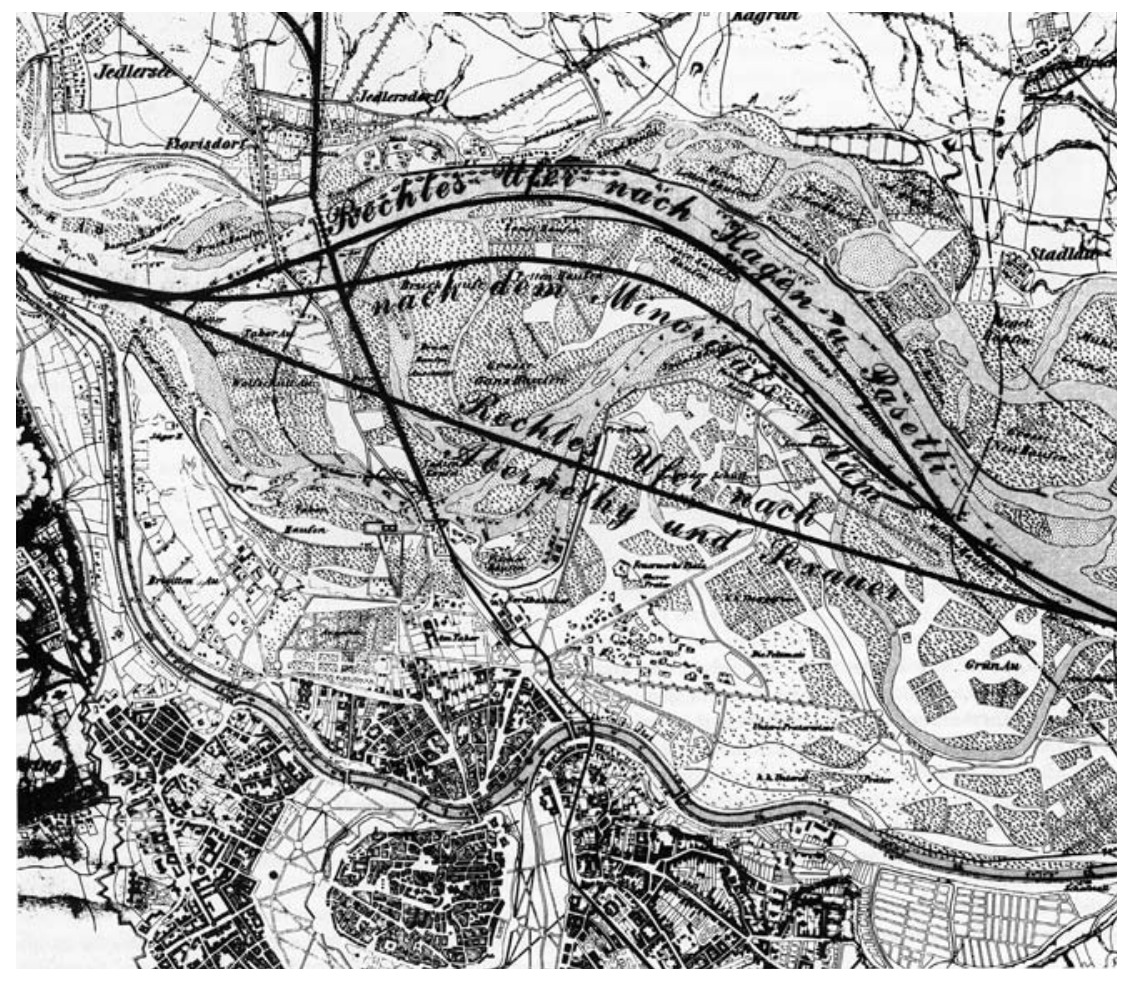

Abb. 3: : Varianten der Donau-Regulierungs-Kommission, nach Hagen und Pasetti (oben) und Abernethy und Sexauer (unten, ausgeführt) (Michelmayr 1994)

die Inland-Navigation nutzen und der Wasserweg insbesondere von Istanbul her wäre kürzer. Mit dieser Publikation hat sich demnach von Wex erstmals mit der Donau auseinander gesetzt, dem Fluss also, der seine künftige Karriere beherrschen sollte. Das Thema des Eisernen Thores nimmt von Wex $(1863,1872)$ nochmals auf und beschreibt dabei die vorzunehmenden Bauarbeiten. Das eigentliche Projekt wird von den österreichischen Experten erst 10 Jahre später besprochen (von Wex 1881 a).

Anonym (1868) bespricht erstmals die Donauregulierung bei Wien. Es wurden vier Experten eingeladen, darunter der Mentor des deutschen Wasserbaus Gotthilf Hagen (1797-1884) und der Kanalingenieur James Abernethy (18141896) von London. Die wichtigste Frage war: Auf welche Art kann die Donau-Regulierung am sichersten erzielt werden? Alle vier Experten waren der Ansicht, dass durch die Herstellung von festen, parallelen und zusammenhängenden Ufern die Aufgabe gelöst wäre, falls die Stromgeschwindigkeit möglichst gleichförmig sei. Eine zweite Frage, falls sich die Experten für die Beibehaltung des gegenwärtigen Donaulaufs bei Wien aussprechen würden, war: Wie würden dann die Arbeiten ablaufen? Es sprach sich niemand klar für die Beibehaltung des damaligen Flusslaufs aus, ebenfalls verwies man auf Detail-Berichte betreffend des Bauablaufs und der Kosten (Abb. 3).

Eine definitive Antwort folgte einige Monate später vom Vorsitzenden Abernethy. Durch die Errichtung von befestigten Dämmen und einen zusammenhängenden Flusslauf wird eine gleichmäßige Fließgeschwindigkeit erzielt. Dabei sollte das Flussprofil aus zwei Teilen bestehen nämlich dem Hauptgerinne und den Vorländern. Starke Kurven sollten möglichst vermieden werden, um das Ufer zu schützen. Dieses Verfahren sollte nicht nur in Wien, sondern entlang der gesamten Donau angewendet werden. Die Kosten wurden auf 2 Mio. damalige englische Pfund veranschlagt, also einem bedeutenden Betrag. Damit waren die zu unternehmenden Arbeiten von den Experten abgesteckt, es galt nun, diese auszuführen. Mit dieser Aufgabe wurde in der Folge insbesondere Oberbaurath Gustav von Wex betraut, der vorerst die Expertenberichte zu würdigen hatte.

In seinem Bericht hielt von Wex fest, dass es wichtig wäre, die Donau bei Wien in einen einzigen Strom zu vereinen, und damit alle Nebenarme zu beseitigen. Ferner sollte ein Normalbett erstellt werden mit parallelen und festen Ufern, welche die Gleichmäßigkeit des Flusslaufs sicherten. Ferner sollte das Querprofil aus einem Mittelbereich sowie aus den Vorländern 


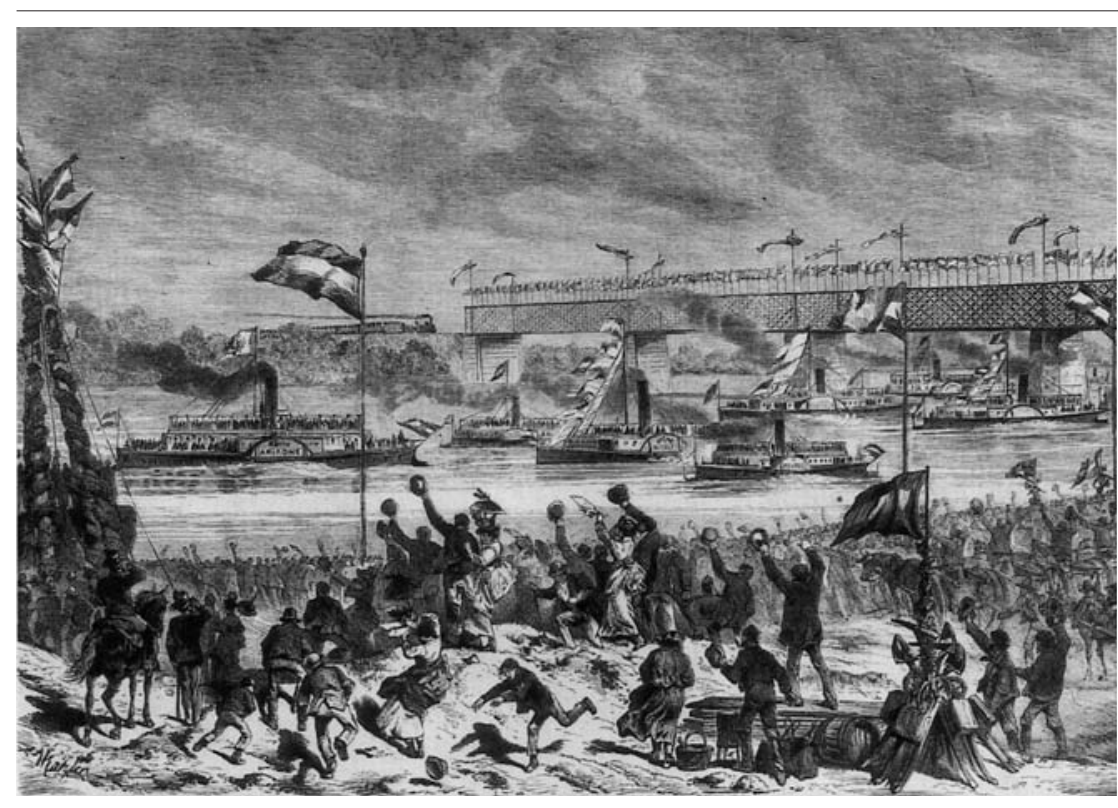

Abb. 4: : Eröffnung der Schifffahrt auf der neuen Donaustrecke bei Wien 1875 (Konold 1994)

bestehen, wie dies von den Experten vorgeschlagen wurde. Damit würde neben dem Hochwasserschutz auch die Schifffahrt insbesondere bei Niederwasser unterstützt. Es wurde weiter festgehalten, dass die Donauregulierung bei Wien nur durch die Führung der Donau mittels eines Durchstichs sichergestellt werden konnte. Weitere Beachtung fanden der Wiener Donaukanal sowie die Hafenbauten für die Hauptstadt.

Die Donau-Regulierung wurde am 14. Mai 1870 feierlich durch Seine Majestät dem Kaiser begonnen, indem er den ersten Spatenstich vornahm (Anonym 1870). An diesem Tag wurde von Wex zum Ministerialrath ernannt. Darauf verlas der Kaiser die folgenden Worte: „Mit wahrer Freude nehme ich Theil an der Feier des heutigen Tages, die einem Unternehmen die Weihe gibt, von dessen glücklichem Gelingen ich mit Ihnen allen die segenreichsten Folgen für die Stadt Wien, das Land Niederösterreich und das ganze Land erwarte. Ich bin den bisherigen Einleitungen für die Inangriffnahme dieses Werkes mit lebhaftestem Interesse gefolgt und habe mit Befriedigung die Opferwilligkeit begrüßt, mit welcher die Landesvertretung und der Gemeinderath von Wien, durchdrungen von der Nothwendigkeit und hohen Nützlichkeit der Donau-Regulierung, zu ihrer Durchführung in gleichem Verhältnisse wie die Staatsfinanzen mitwirken. Für die so rasche Einleistung der Arbeiten spreche Ich den Mitgliedern der Donau-Regulierungs-Commission Meine vollste Anerkennung aus. Ihre Umsicht und Tathkraft, sowie die erprobt
Tüchtigkeit der Unternehmer flösst mir das Vertrauen ein, dass die in das Werk gesetzten Hoffnungen sich im vollen Umfange verwirklichen werden. Der Himmel gebe ihm seinen Segen.“

Bereits ein Jahr später veröffentlichte von Wex (1871 a, b) einen ersten Bericht zu den laufenden Bauarbeiten. Nach einer historischen Einleitung zur Donau in der Umgebung von Wien wird insbesondere das Projekt von Joseph Schemerl (1754-1844) um 1810 zur Hochwassersicherheit besprochen, welches aber nicht ausgeführt wurde. Nach den Hochwassern von 1849/50 und 1862 wurde die Sache nochmals angegangen, die schliesslich in den erwähnten Expertenbericht von Abernethy und anderen mündete. Es wird ferner auf den beachtlichen Donau-Aufstau bei Nussdorf infolge von Spornen und falsch angelegten Dämmen hingewiesen. Weiterhin schlägt von Wex vor, die Donaustrecke zwischen Nussdorf und Albern zu begradigen, um damit die Schifffahrt zu verbessern sowie die Hochwassergefahr durch beidseitige Ufereindämmung zu beseitigen. Die Kosten dieses Projekts sollten je zu einem Drittel vom Staat, vom Lande Niederösterreich und von der Stadt Wien getragen werden, wie dies auch vom Kaiser vorgeschlagen wurde.

Eine weitere Frage betraf das FlussNormalprofil. Die Donau hat einen Normalwasserdurchfluss bei Nussdorf von etwa $1200 \mathrm{~m}^{3} / \mathrm{s}$, während bei Hochwasser rund $7300 \mathrm{~m}^{3} / \mathrm{s}$ anfallen. 1862 erreichte der Durchfluss sogar knapp $10000 \mathrm{~m}^{3} / \mathrm{s}$. Infolge dieses großen Durchfluss-Spektrums wäre es ungeeignet, einen ein-profi- ligen Querschnitt zu wählen. Schließlich wurde ein zusammengesetztes Querprofil vorgeschlagen, dessen Mittelwasserbereich eine Breite von gut $280 \mathrm{~m}$ und der Vorlandbereich von insgesamt $475 \mathrm{~m}$ aufweist. Weitere Beachtung fanden der genaue Wasserspiegelverlauf, die Anordnung der verschiedenen Brücken, die Zukunft des Wiener Praters sowie die Anlegung der Eisenbahnen entlang der Donau. Es wurde auch die Rentabilität des gesamten Donauwerks besprochen. Die etwas über $13 \mathrm{~km}$ lange Regulierungsstrecke von Nussdorf bis Albern wurde mi zwei Durchstichen bewältigt: Der obere, $6,6 \mathrm{~km}$ lange Durchstich von Floridsdorf bis zur Stadlauer Ostbahnbrücke wurde in der ganzen Flussbreite und in der vollen Tiefe ausgehoben, während für den unteren Durchstich eine „Künette“ gegraben und der Rest dem fließenden Wasser überlassen wurde. Das beauftragte Bauunternehmen Castor, Couvreux und Hersent brachte einen grossen Teil der Baumaschinen vom Suezkanal, der kurz vorher eröffnet wurde.

Die Donauregulierung bei Wien kann nach von Wex (1876 a) zu den wichtigsten und großartigsten Bauanlagen der Neuzeit gezählt werden (Abb. 4). In einem Vortrag erklärt er Mitgliedern des Österreichischen Ingenieur und Architekten-Vereines die aktuellen Arbeiten und konstatiert, dass diese auch den jüngsten Hochwässern standgehalten haben. Es werden ferner auch zwei Pläne vorgestellt, der eine aus dem Jahr 1706 nach Anguissola, Marinoni und Steinhauss mit eingezeichnetem neuem Flusslauf, der andere als aktueller Plan der Donau um Wien vor und nach der Regulierung. Weitere Angaben, insbesondere betreffend der Donau-Eisführung, folgten durch von Wex (1876 b).

Von Wex (1880 b) berichtet über die Fortschritte der Donauregulierung be Wien (Abb. 5). Er stellt sich die Frage: Entsprechen die eingeleiteten Arbeiten anerkannten hydrotechnischen Grundprinzipien, und haben sich die erwarteten Vorteile dieses Projekts eingestellt? Nach den drei Hochwassern der Jahre 1876, 1877 und 1879 sind Fluss-Querprofile rund alle $100 \mathrm{~m}$ ermittelt worden. Aus den Quer- und Längsprofilen zwischen Nussdorf und der verlängerten Kanal-Ausmündung, also der bis 1880 fertig gestellten Korrektionsstrecke, lässt sich folgendes festhalten: Die mittleren Wassertiefen haben in den 5 Jahren von 3,3 auf 3,5 $\mathrm{m}$ zugenommen und die Donau hat sich durch die Arbeiten vergleichmäßigt. Ablagerungen werden durch die Hochwässer wieder ausgewaschen, wo- 

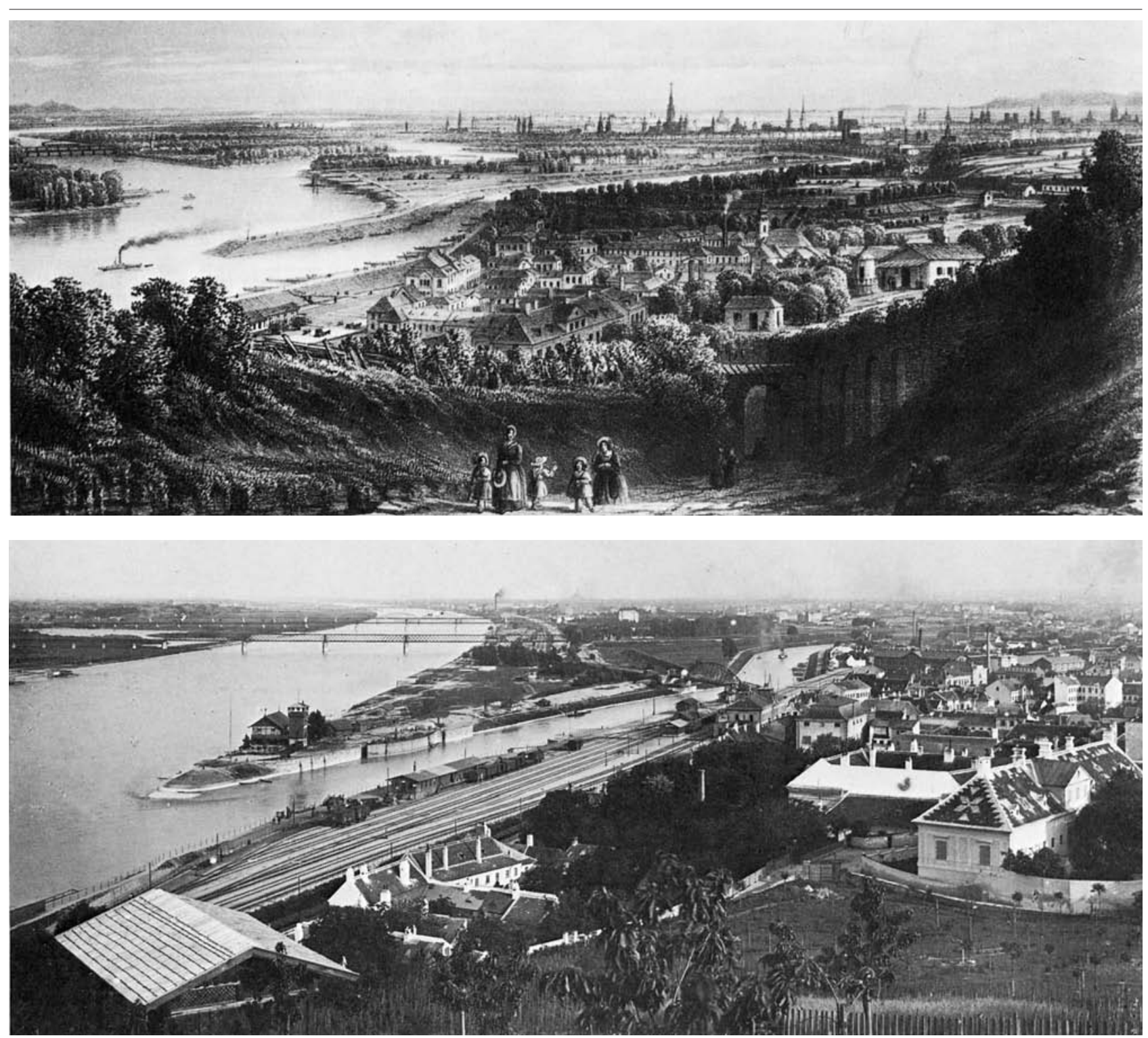

Abb. 5: Donau von Nussdorf aus gesehen vor (oben) und nach (unten) der Regulierung, mit Schwimmtor und Bahn (Buchmann et al. 1984)

mit sich ein langfristiger Gleichgewichtszustand einstellen sollte.

Es wurde weiterhin festgestellt, dass durch die neue Profilgeometrie die Ufe auch nach den Hochwassern unbeschädigt blieben und dass weitere Bauwerke entlang der Donau ebenfalls keinen Schaden erlitten hätten. Schließlich habe das Überschwemmungsrisiko der Stadt Wien abgenommen, das Marchfeld sei nicht mehr wie früher überschwemmt worden, und die Eisstösse hätten ihre Gefährlichkeit verloren. Die Schifffahrt sei selbst bei den niedrigsten Wasserständen nie beeinträchtigt gewesen. Der Erfolg der DonauKorrektion um Wien sei auf ähnlichen Werken basiert, wie sie etwa André Baum garten (1809-1859) an der Garonne in Frankreich zum ersten Mal wissenschaft- lich um 1846 verfolgt habe und dann durch Grebenau am Rhein zwischen Strassburg und Kehl in den 1860er Jahren fortgesetzt habe. von Wex hat ebenfalls alternierende Geschiebebänke auf der Donau festgestellt, wie dies vor ihm seine zwei Kollegen aus Frankreich und Deutschland beobachtet hätten. Abschließend weist er auf die großen wirtschaftlichen und sozialen Vorteile dieses Projekts hin, welches schon nach wenigen Jahren finanziell gesehen positiv abschließen würde. Weitere Schriften über die Wiener Donauregulierung im speziellen und den Wasserbau Österreichs sind von Baumann (1951) zusammengestellt worden. Einen Überblick über die historische Wasserwirtschaft an der Donau gibt ebenfalls Michelmayr (1994).

Es kann abschließend festgehalten werden, dass die Donau-Regulierung bei Wien keine lange Fließstrecke beinhaltete, dass es sich aber um den größten Fluss Westeuropas handelte, der bis anhin reguliert wurde. Hält man sich die Tatsachen vor Augen, dass um diese Zeit etwa die ersten Normalabflussbeziehungen der Hydraulik ermittelt wurden und gleichzeitig über den Sedimenttransport, den Abfluss in komplizierten Profilen oder die Langzeitentwicklung einer Sedimentsohle praktisch nichts bekannt war, so muss festgestellt werden, dass der Erfolg dieses Projekt insgesamt als sehr positiv betrachtet werden darf. Dieser Erfolg fußt auf den von der Expertenkommission festgehaltenen Grundsätzen, insbesondere der Wahl des geeigneten Querprofils, der Umgestaltung des Einlaufbereichs bei Nussdorf und 
der Vereinigung des Flusses in einen Hauptlauf. Vom heutigen Standpunkt aus mögen gewisse Grundsätze einen anderen Akzent erhalten haben, wesentlich ist jedoch, dass mit der Donau-Regulierung der Stadt Wien ein bedeutend höherer Hochwasserschutz gegeben werden konnte. Dieses Verdienst kann von Wex zu einem bedeutenden Teil für sich verbuchen.

\subsection{Hydraulik}

Von Wex (1864) wird sich mit dem Titelauszug „Unrichtigkeit der bisher gebrauchten hydraulischen Formeln" be seinen Kollegen kaum viele Freunde gemacht haben. Er hält fest, dass die Hydraulik während der letzten Dezennien wenige Fortschritte etwa im Vergleich zur erfolgreichen Industrialisierung gemacht habe. Besonders arg sei es bei den Flussregulierungen, da Überschwemmungen große Schäden bei der Bevölkerung, der Schifffahrt und den Agrikulturen anrichteten. So sollen die damals vorhandenen Lehrbücher voneinander stark abweichende Meinungen betreffend der richtigen Flusskorrektionen vertreten, was zu beträchtlichen Problemen führe. Er bezieht sich dann auf die ",alten Hydrauliker“ Belidor, Silberschlag, Schemerl, Wiebeking, Woltman und Pechmann, die mit Buhnen gute Resultate bei Flüssen erzielt haben sollen. In letzter Zeit sollen jedoch Buhnen in Misskredit geraten sein, da durch diese grosse Schäden angerichtet würden, so etwa auf der Loire, da die Buhnen zu weit voneinander entfernt wären. Obwohl der berühmte Wasserbauer Gotthilf Hagen (1797-1884) um 1853 Versuche mit Buhnen angestellt habe, wisse man nicht, ob Buhnen in Flüssen angewendet überhaupt sinnvoll wären. Weitere Unsicherheiten seien bei Stromdurchstichen zu erwarten, wo wiederum wenige Angaben in der einschlägigen Literatur zu finden seien. Von Wex tritt dann auf die damals jüngsten Forschungsresultate der beiden Amerikaner Humphreys und Abbot ein, welche 1861 großangelegte Naturversuche am unteren Mississippi vornahmen, um die Hydraulik von Flüssen zu erforschen.

Dann kommt von Wex auf die Ermittlung des Durchflusses mittels vollkommenen Überfällen zu sprechen. Mittels der damals bekannten Formeln erhält er für ein Berechnungs-Beispiel große Abweichungen. Diese werden beim unvollkommenen Überfall noch bedeutender Zusammenfassend lässt sich feststellen, dass damals wie auch heute von der For- schung vielleicht mehr erwartet wird, als kurzfristig zu leisten ist. Buhnen sind auch heute noch weitgehend unbekannt hinsichtlich ihrer Wirkung auf eine Flussstrecke. Der Grund dazu liegt in der Komplexität der räumlichen Strömung, der Vielzahl der maßgebenden Parameter und der Zweiphasenströmung Sediment-Wasser. Häufig sollten zudem Antworten zu biologischen oder morphologischen Fragen beantwortet werden, was die heutigen Kenntnisse übersteigt. Betreffend Überfällen wurden bereits im 19. Jahrhundert, insbesondere durch Henry Bazin (18291917), die meisten Antworten durch eine minuziöse Beobachtung gegeben, was schließlich zu einer befriedigenden Lösung beitrug. Dieses rein hydraulische Problem wurde schließlich durch Theodor Rehbock (1864-1950) um 1930 abschließend gelöst.

Liest man diese Publikation, so fühlt man sich in eine andere Zeit versetzt: Heute wird mit Renaturierungsarbeiten praktisch das Gegenteil dessen verfolgt, was vor 140 Jahren als fortschrittlich galt. Die meisten damaligen Grundsätze für eine „Flusskorrektion“ stehen demnach diametral zur heutigen Maxime. Ohne hier auf die Argumentation einzugehen, lässt sich auch beim Flussbau ein stetiger Trendwechsel beobachten. Man kann weiter fragen, wie lange es gehen wird, bis die heutigen Regeln wieder veraltet sind Die Leidtragenden sind einerseits die Natur selbst, mit der solche Prüfungen angestellt werden, andererseits sicher aber auch die Steuerzahler, die diesen Fortschritt zu bezahlen haben.

Von Wex (1873) geht der Fragestellung nach, ob die Flüsse Mitteleuropas zeitlich immer weniger Abfluss erhalten infolge von hydrologischen Entwicklungen. Diese wohl gewagte Behauptung will er mittels einer Datenanalyse unterstützen. Dabei geht er von Pegelbeobachtungen aus, die unter anderem von den bedeutenden deutschen Hydrographen Friedrich Hagen (1829-1892), dem Sohn Gotthilfs, oder Heinrich Grebenau (18221877) erhoben wurden. Die darin untersuchten Flüsse betreffen die Elbe, die Weichsel und die Donau bei Wien. von Wex weist dabei auch auf den Einfluss von zukünftigen Arbeiten hinsichtlich Stromregulierungen, Schifffahrtskanäle, Wasserleitungen und der Wasserversorgung im Allgemeinen hin. Er glaubt auch festgestellt zu haben, dass trotz ständig kleinerer Durchflüsse die Hochwassergefahr sich in den letzten Dezenien vergrößert habe, dies insbesondere infolge der Abholzung vieler Wälder. Um ein ausgeglicheneres Abfluss-Regime zu schaffen, wären große Geldsummen nötig, die jedoch letztlich einem Lande, seiner Bevölkerung und den NutzerInnen eines Flusses zugute kämen. Solche Veränderungen ließen sich freilich nur durch Gesetzesänderungen herbeiführen, der Staat hätte also in Zukunft ein bedeutendes Wort in der „Bewirtschaftung von Flüssen" mitzureden.

Als Antwort auf von Wex' (1873) geschilderten Ausführungen gibt das hydrotechnische Comité mit Deutsch (1875) als dessen Obmann die folgende Stellungnahme. Das Comité bestätigte grundsätzlich die anhand der Zahlen gemachten Aussagen von Wex', es wurden aber Bedenken hinsichtlich dessen Argumentation geäußert. Die Kritik betrifft dabei die sich laufend verändernden Flussläufe, die Verschiebungen von Pegelstationen und deshalb die gesamte Flussdynamik. Es werden zudem zwei wichtige Fragen aufgeworfen: (1) Ist die größere Hochwasserfrequenz und die verminderte Niederwasserhöhe nur eine Erscheinung in Deutschland und Österreich, oder kann diese Tendenz auf alle „entwickelten Staaten“ übertragen werden?, und (2) Was sind die Ursachen eines solchen Regimes, und welche Maßnahmen müßten ergriffen werden? Die erste Frage wird positiv beantwortet in dem Sinne, dass diese Entwicklung nicht nur in deutschsprachigen Gebieten eingetreten ist. Betreffend der Frage 2 werden eine Vielzahl von möglichen Ursachen für eine erhöhte Hochwassergefährdung genannt. Häufigere Niederwasserzustände werden demnach erzeugt durch erhöhten Wasserverbrauch Austrocknung von Sumpflandschaften, Abnahme der mittleren Niederschläge, und durch die Abholzung der Wälder, welche einen geringeren Retentionseffekt erzeugen. Es werden schließlich die folgenden Schlüsse gezogen: (1) Die hydraulischen Abflüsse in Flüssen haben sich in der vergangenen Zeit in der Tat vom Mittelwerten weg zu Extremen bewegt, mit mehr Nieder- und Hochwasserperioden als noch vor einem Jahrhundert und (2) die Ursachen sind hauptsächlich in der ,Devastation der Wälder zu suchen, neben den bereits angefügten Gründen Schließlich werden die folgenden Forderungen der Regierung unterbreitet: (1) Flüsse sollen mit regelmäßigen Profilen ausgebildet werden, (2) Wasserkarten mit Längsprofilen aller wichtigen Flüsse sollen ausgeführt werden, (3) Wälder sollen unter keinen Umständen kahlgeschlagen werden, (4) Wälder dürfen nur noch in 
Niederungen "ausgerodet“ werden, (5) Kulturpläne dienen zur Beurteilung von Agrikulturen, (6) Anlegung von Schirmpflanzungen in baumlosen Ebenen, (7) Schaffung von Reservoiren bei Flussregulierungen und Entsumpfungen, (8) Ströme sollen systematisch und einheitlich schiffbar gemacht werden, und (9) Schifffahrt und Eisenbahn sollen in gleichem Grade gefördert werden. Abschließend lässt sich anmerken, dass die Visionen im Flussbau des ausgehenden 19. Jahrhunderts speziell von Frankreich ins Leben gerufen wurden, wo Alexandre Surell (1813-1887) bereits um 1840 die Regierung mittels einer Denkschrift aufforderte, den allgemeinen Schutz von Wäldern anzuordnen. Seine ersten Erfolge verzeichnete Surell jedoch erst kurz vor dem Tode, als alle Alpenländer seine Ideen in Gesetze umzuwandeln begannen. Damit wurde eine Voraussetzung für den modernen Flussbau geschaffen, der zweite in der Form von Fluss-Renaturierung hatte freilich noch rund 100 Jahre zu warten.

Von Wex $(1879,1880$ c, 1881 b, 1882 1891) griff dasselbe Thema nochmals auf. Vorerst weist er auf das rege Interesse an seinen Ausführungen von einer Anzahl nationaler und internationaler Kollegen hin. In den vergangenen 6 Jahren hätte er weitere Untersuchungen angestellt, die seine Vermutungen untermauerten. Das Ziel der vorliegenden Arbeit sei es, einerseits die erhaltenen Diskussionen zu kommentieren und andererseits mit neuen Daten die ursprüngliche Annahme abzustützen. Vorerst werden 22 Berichte zur Arbeit von Wex' erläutert, die alle das Grundphänomen bestätigen, jedoch eine weitere Differenzierung der Tatsache wünschen. Hingegen verwerfen 8 Autoritäten auf diesem Gebiete die Ansichten von Wex.' Als wesentliche Gründe werden genannt: (1) Die erhobenen Daten bezogen sich zum Teil auf Flüsse, die bereits reguliert worden sind oder deren Bette sich vertieft haben, (2) Aus den Wasserstandsabnahmen kann nicht direkt au eine Durchflussabnahme geschlossen werden, (3) Aus den Extremen der Wasserführung kann nicht auf eine generelle jährliche Wasserabnahme geschlossen werden, (4) Mindestens 200-jährige Beobachtungen einer Ganglinie wären erforderlich, (5) Nur mittels jährlichen Durchflussbeobachtungen könnte eine generelle Durchflussreduktion bewiesen werden (6) Noch kein definitiver Zusammenhang bestehe zwischen Waldrodungen und Niederschlagsintensitäten, und (7) Flüsse vertieften ihr Bett im Laufe der Zeit, womit
Pegelbeobachtungen allein ungenügend seien. Die Einwendungen von Heinrich Grebenau (1822-1877) werden dabei speziell besprochen.

Abschließend darf festgehalten werden, dass von Wex mit diesen Untersuchungen quantitativ wenig erreicht hat, dass er jedoch im deutschsprachigen Raum viele seiner Kollegen aufgerafft hat zu einer - nach heutigem Sprachgebrauch - nachhaltigeren Bewirtschaftung von Flusslandschaften. In der Tat sind dann etwa in der Schweiz des ausgehenden 19. Jahrhundert Wälder gesetzlich geschützt worden, da ihr großer Einfluss auf den hydrologischen Abflussprozess erkannt wurde.

Das „wissenschaftliche Vermächtnis" von Wex' ist sicherlich seine Hydrodynamik (von Wex 1888). Der volle Zusatz zum Titel lautet „Entwicklung neuer genauer Formeln zur Berechnung der Wasserabflussmengen bei Überfallwehren, Grundschleusen, Schützenöffnungen und bei Wasserausleitungen in Canäle, ferner Mittheilungen über die neuesten, diesbezüglich in Amerika, in Österreich und in Italien im großen Massstabe durchgeführten Versuche." Unter seinem Namen finden sich die folgenden Titel „k. k. Hofrath und Oberbauleiter der Donau-Regulirung bei Wien, Ritter des k.k. österr. LeopoldOrdens, des kais. Russ. Annen- und des kön. ital. Kronen-Ordens II Classe, Mitglied mehrerer wissenschaftlicher Vereine“. Das Buch ist in 24 Kapitel unterteilt, deren Überschriften alle mindestens 2 Zeilen Länge aufweisen. Grundsätzlich können die folgenden Gebiete ausgemacht werden: Ausfluss und Überfall, Durchfluss und Schützen, Durchflussmessung, Tafeln.

Im Vorwort verweist von Wex nochmals auf seine bereits 1864 gemachten Äusserungen betreffend der zum Teil großen Abweichungen verschiedener Formeln auf ein bestimmtes Abflussproblem. Da er zwischen den Jahren 1868 bis 1879 vollauf mit der Projektierung und Oberbauleitung der Donauregulierung bei Wien in Anspruch genommen wurde, hätte er erst ab 1880 sich wieder hydraulischen Problemen zuwenden können. Dabei hätte ihm das Buch von Rühlmann (1879) große Dienste geleistet. Das Buch Hydromechanik kann weder als Lehrbuch noch als eine Zusammenfassung bestehenden Wissens betrachtet werden, noch ist es vergleichbar mit einem Werk das dem praktizierenden Ingenieur etwa direkt Hilfe leistet, da oft bekannte Vorschläge nur kritisiert werden, um dann ein

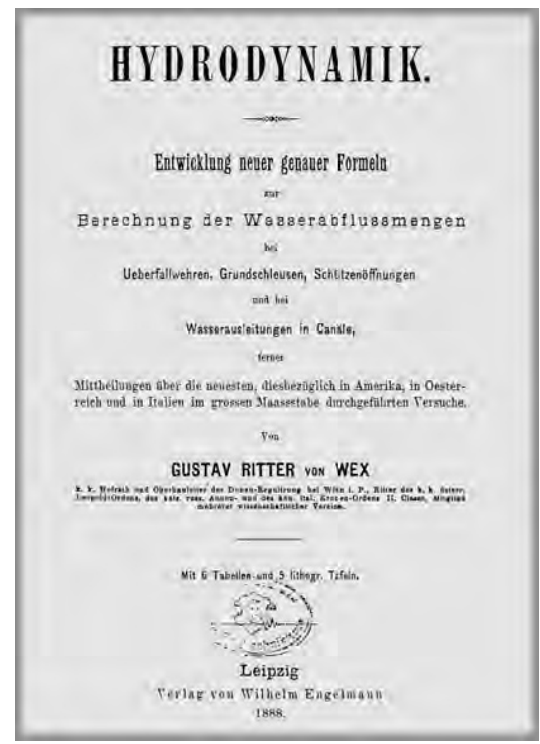

Abb. 6: Titelblatt von Wex (1888)

„ohne Anwendung irgendeiner Hypothese und nur mit genauer Beachtung der als richtig erkannten Grundprinzipien der Hydrostatik und Hydrodynamik neue hydraulische Formeln analytisch zu entwickeln". Leider hat es aber von Wex unterlassen, seine neu entwickelten Formeln mit ausgewählten und damals bereits vorhandenen Messwerten zu verifizieren. So bleibt seine im Buchtitel gemachte Aussage im Vakuum stehen.

Der Einfluss der Zuflussgeschwindigkeit von Wehren oder Durchlässen etwa kann natürlich in den Formelapparat eingebaut werden, nur werden dadurch die Ausdrücke zu kompliziert, und der entsprechende Beiwert müsste auch noch je nach Geometrie bekannt sein. Heute nimmt man etwa für den Ausfluss aus Schützen die einfachste Form $Q=\mu F(2 g H)^{1 / 2}$ an mit $Q$ als Durchfluss, $\mu$ als Kontraktionsbeiwert, $F$ als Ausflussfläche, $g$ als Erdbeschleunigung und $H$ als die wirkende Energiehöhe. Der gesamte Kontraktionsvorgang ist deshalb im Beiwert $\mu$ enthalten. Solche Werte spezifiziert jedoch von Wex in seinem Buch nicht. Die Berechnung von Stau- und Senkungskurven wurde in Frankreich bereits um 1830 vorgeschlagen, von Wex geht jedoch auf ein primitives Verfahren zurück, wiederum ohne jegliche Diskussion anderer Verfahren.

Beim Thema Normalabfluss werden die Beziehungen von Darcy-Bazin verwendet, jedoch wieder mit einer entsprechenden Kritik des Werks dieser außerordentlichen Hydrauliker des 19. Jahrhunderts. Von Kutter oder anderen Kollegen fällt in diesem Zusammenhang 
kein Wort. Der Titel des Buchs ist falsch gewählt, handelt es sich doch um Hydraulik und keineswegs um die stark mathematisch geprägte Hydrodynamik. Das Buch enthält keine Referenzen, die Bilder sind auch nicht im Textteil enthalten, sondern als Tafeln am Buchende angelegt, eine damals verschwindende Technik im Buchwesen. Insgesamt hätte man das Buch besser mit Überfall, Ausfluss und Durchfluss betitelt, da eigentlich nur diese drei Themen angeschnitten werden. Ich kann mir persönlich nicht vorstellen, dass dieses Werk einen großen Einfluss auf die Hydrauliker von damals hatte, da es um einiges bessere Bücher in diesem Fachgebiet gab.

\section{Schlussfolgerungen}

Das Leben und Wirken einer markanten Persönlichkeit des 19. Jahrhunderts, Gustav Ritter von Wex, wird vorgestellt mittels seiner Biografie, seiner großen Arbeiten zur Donau-Regulierung bei Wien, seiner Publikationen und seines Buchs Hydromechanik. Dabei ist von Wex' Anteil an der Donau-Regulierung sicherlich außerordentlich. Wie aus seinen Schriften jedoch hervorgeht, hat sich das Wesen des Flussbaus in den vergangenen 140 Jahren komplett verändert. Die wesentlichen damals gesetzten Ziele wurden mit diesem mit viel Interesse verfolgten Projekt jedoch erreicht. Der beachtliche Wandel betref- fend Flussbauten und Hochwasserplanung zwischen 1870 und 2010 wird aufgezeigt mit der Frage, wo es denn in der Zukunft hingehe.

\section{Danksagung}

Ich möchte mich bei Herrn Dr. Michael Pfister, VAW, für die Durchsicht und seine Anmerkungen zu dieser Arbeit freundlichst bedanken.

\section{Korrespondenz: \\ Prof. Dr. Willi H. Hager \\ VAW, ETH-Zürich}

hager@vaw.baug.ethz.ch

\section{LITERATUR}

Im Folgenden wird mit ÖZIV die „Zeitschrift des Österreichischen Ingenieur-" und Archides Osterreichischen Ingenieur-

Anonym (1868) Die Donauregulierung bei Anonym (1868) Die Donauregulierung bei 20(9/10), 173-192.

Anonym (1870) Inaugurirung der Donau-Regulierungs-Arbeiten. ÖZIV 22(45), 105-106. Anonym (1876) Gustav Ritter von Wex. ÖZIV 28(8), 64; 28(45), 300 .

Anonym (1881) Gustav Ritter von Wex. ÖZIV 33(26), 170

Anonym (1892) Hofrath Gustav Ritter von Wex. OZIV 44(41), 526.

Baumann F (1951) Vom älteren Flussbau in sterreich. Schriftenreihe des OsterreichiSpringer, Wien.

(1984) Der BM, Sterk H, Schick

(1984) Der Donaukanal: Geschichte, Planung, Ausführung. Beiträge zur Stadtforschung Astoria, Wien

Deutsch J (1875) Bericht des hydrotechnischen Comité's über die Wasserabnahme in den Quellen, Flüssen und Strömen. ÖZIV 27(8/9), 157-165.

Kleinschroth A (1992) In Memoriam: Gustav von Wex - Zum 100. Todestag. Wasser und Boden 44(9), 584

Künster R, Jarosch W (1935) Die Wiener Wasserbauten. Festschrift herausgegeben anlasssich der Hundertjaht heier des Wiene uur Jugend und Volk, Wien.

Michelmayr $F(1994)$ Geschichte der Donauregulierung in Wien. Historische Wasserwirt566, W. Konold, ed. Wittwer, Stuttgart. Roeder R (1892) Hofrath Wex. Centralblat der Bauverwaltung 12, 456 . Rühlmann M (1879) Hydromechanik oder die technische Mechanik flüssiger Körper. Hahn, Hannover.
Wex G von (1857) Darstellung der physischen Schifffahrtshindernisse an der Ausmündung des Donaustromes ins Schwarze Meer und Mittel zu Wex G von (1863) Der Donaustrom als Hauptverkehrsstrasse nach dem Orient, nach erfolgte Beseitigung der Schifffahrtshindernisse am Eisernen Thore und nach den sieben Stromschnellen oberhalb Orsova. Österreichische Revue 3, 68-122.

Wex G von (1864) Über den gegenwärtigen Stand der Wasserbauwissenschaft und die Unrichtigkeit der bisher gebrauchten hydraulischen Formeln. Bericht über die 14. Versammlung

31. August und 1., 2. September 1864, 124-133. 31. August und 1., 2. Sep

Wex $G$ von (1871 a) Die. Wex G vits ( 1871 a) Die Donauregulierung bei 430-438; 15, 506-518.

Wex G von (1871 b) Die Donauregulierung bei Wien. ÖZIV 23(7), 147-151; 23(8), 157-164. Wex G von (1872) Über die Schiffbarmachun der Donau am Eisernen Thore und an den sieben Felsbänken oberhalb Orsova. ÖZIV 24(10), 281-290.

Wex G von (1873) Über die Wasserabnahme in den Quellen, Flüssen und Strömen [bei gleichzeitiger Steigerung der Hochwässer in den Culturländern]. OZZIV 25(2), 23-30; 25(4), 63-76; 25(6/7), 101-119. Ebenfalls erschienen leicht abgeandert in Schitten des Vereines zur Verbrei1874/75 15,323 362 . Wex G von (1876 a) Die Wiener Donauregulieung. Schriften des Vereines zur Verbreitung naturwiss

Wex G von (1876 b) Über die Wirkungen der bisher ausgeführten Donau-Regulierungsarbeiten auf die Beseitigung der Uberschwemmungsgefahren bei Wien. OZIV 28(5), 77-88. Wasserabnahme in den Quellen, Flüssen und
Strömen, bei gleichzeitiger Steigerung de Hochwässer in den Culturländern. ÖZIV 31(6/7),

nauregulierung anlässlich de Wirkung der DoWinters und der Hochwässer im August 1880. Waldheim \& Förster, Wien.

Wex G von (1880b) Über die Fortschritte der Ausbildung des neuen regulierten Donau-Strombettes bei Wien und über die hierbei gemachten Erfahrungen. OZIV 32(3), 37-43.

Wex G von (1880c) Second treatise on the decrease of water in springs, creeks and rivers contemporaneously with an increase in height of floods in cultivated countries, Ubersetzung vo shington DC

Wex G von (1881a) Diskussion über die Frage der Regulirung der Stromschnellen auf frage der Regulirung der Stromschnellen auf der unte110; 33(15), 115-117; 33(16), 123-128; 33(17), 131-136; 33(36), 209-211.

Wex G von (1881b) Entgegnung auf die Broschüre des Geheimen Ober-Baurathes Herrn Dr. G. Hagen: Uber Verănderung der Wasserstände in den preussischen Strömen. Waldheim \& Förster, Wien.

Wex G von (1882) Technisches Gutachten über die projectirten Wasserbauanlagen und über den Zollcanal aus Anlass des Anschlusses Hamburg an das deutsche Zollgebiet. Waldheim \& Förster, Wien

Wex G von (1888) Hydrodynamik, Entwicklung neuer genauer Formeln zur Berechnung der Wasserabflussmengen bei Uberfallwehren, Wasserausleitungen in Canäle. Engelmann, Leipzig.

Wex G von (1891) Periodische Meeresanschwellungen an den Polen und Äquator, hiedurch veranlasste Uberflutungen der Polar- und Aquatorial-Länder, dann Sintfluthen, Eiszeiten und Vergletschungen der Alpen. Spielhagen \& Schurich, Wien 